\title{
Field Screening of Soils Contaminated with Explosives Using Ion Mobility Spectrometry

$$
\begin{aligned}
& \text { INEL/CON-97-00085 } \\
& \text { CONF-970113--5 }
\end{aligned}
$$

David A. Atkinson, Alan B. Crockett, Idaho National Engineering Laboratory, Lockheed Martin Idaho Technologies Company, P.O. Box 1625, Idaho Falls, ID 83415-2213 and

Thomas F. Jenkins, U.S. Army Cold Regions Research and Engineering Laboratory, 72 Lyme Road, Hanover, NH 03755-1290

\begin{abstract}
This study involved the comparison of IMS screening with EPA's standard method for explosives, Method 8330. The U.S. Army Corps of Engineers provided a large number of soil samples that had been collected from three locations at each of three explosive contaminated installations. The samples had been dried, ground, homogenized and analyzed in duplicate by Method 8330. Duplicate two gram aliquots of these samples were extracted with $10 \mathrm{~mL}$ of acetone by shaking for three minutes, allowed to settle, then analyzed by IMS for Method 8330 compounds. Half of the extracts from one location have also been analyzed in duplicate by IMS for TNT. Results from TNT contaminated soils look extremely promising. Correlation between IMS and EPA Method 8330 results was very high ( $r=0.99)$. Based on these results, the intention is to further develop and evaluate IMS for simultaneously quantifying multiple analytes. IMS throughput and cost per sample makes it an attractive technique. The ultimate objective is to provide adequate validation data to EPA for inclusion of the method as a screening procedure in SW-846.
\end{abstract}

\section{INTRODUCTION}

Soils contaminated with explosives constitute a high priority problem at some Department of Energy facilities and many Army installations. Because explosives in soil are often heterogeneously distributed and require high density sampling, field screening is essential to characterize sites more quickly, economically and accurately. Current immunoassay and colorimetric field screening procedures have proven useful, but have significant per sample costs and limited throughput. Often, only a single analyte or analyte group determination is possible per sample. At present, several field screening procedures are available for TNT in soil, three procedures for RDX and one procedure for 2,4-DNT and ammonium picrate/picric acid (AP/PA). Ion mobility spectrometry has been used for several years in law enforcement work to detect explosives in air at ppt levels, but limited work has been done to apply the technique to quantifying explosives in soils. IMS offers great potential since many compounds (TNT, RDX, PETN, DNT, TNB, NG, etc.) can be quantified simultaneously in an acetone extract within a few seconds and at a cost of under a dollar/sample.

A study was conducted by Jenkins et al. (1996) to assess the extent of short-range heterogeneity present in contaminant concentrations for surface soils at explosives contaminated sites. Intensive sampling was conducted over short distances. Discrete and composite samples were analyzed by both colorimetric field screening techniques (EnSys) and standard laboratory protocols (EPA Method 8330). Three locations were sampled at each of three installations, duplicate samples were analyzed by each method and the results used to estimate the relative contributions of analytical error and sampling error to the total uncertainty associated with sample collection and analysis for explosives residues. The major contaminant at seven of the nine sampling locations was TNT, and the colorimetric field screening method provided results that were in agreement with laboratory results using SW846 Method 8330. The results of this work have been presented in a formal report and summarized in the proceedings of this meeting.

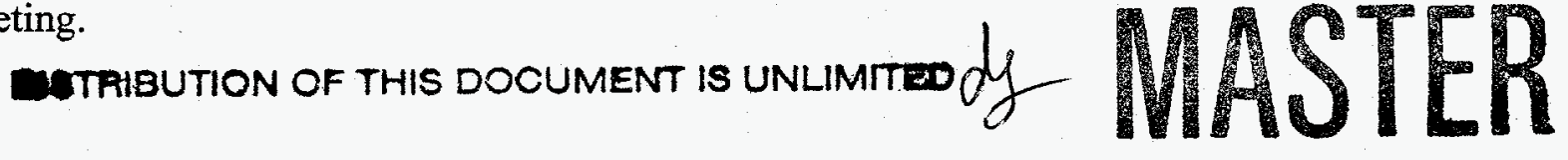




\section{DISCLAMERR}

Portions of this document may be illegible in electronic image products. Images are produced from the best available original document. 
Samples from this study were archived and subsequently made available by CRREL and the U.S. Army Environmental Center for use in evaluating ion mobility spectrometry (IMS) as a field screening technique for explosives in soil. IMS holds considerable potential as a screening method since the instrument can simultaneously detect multiple compounds in acetone extracts of soil. IMS has long been used for forensic purposes to detect extremely low levels of explosives and chemical warfare agents in air. Relatively little work has been done to quantify explosives in soil and we know of no published reports comparing IMS screening results with EPA Method 8330 (Avolio et al. 1995, Rodacy and Leslie 1992)

The objective of this study has been to evaluate the utility of IMS as a screening technique for quantifying explosive residues in soils.

\section{Methods and Materials}

The instrument used for this work has been a PCP 110 equipped with an microliter syringe compatible injection port. While this instrument can be transported to the field, it is not hand portable and the current software is inadequate for simultaneous real time quantification of multiple explosives. The $2 \mathrm{~g}$ soil samples were extracted in batches of 5 to 6 by shaking with $10 \mathrm{~mL}$ of laboratory grade acetone.

\section{Results}

CRREL provided duplicate homogenized $2 \mathrm{~g}$ soil samples from the sites they had previously characterized. The initial effort was to develop a calibration curve for TNT and analyze a composite sample from each of the nine locations sampled by Jenkins. The effort verified that the IMS could instantaneously detect the presence of TNT, DNT and TNB in the samples. Efforts aimed at developing a linear calibration curve demonstrated that injecting varied volumes of a standard resulted in deviations from linear behavior in calibration. Apparently, larger volumes of acetone complicates the ion chemistry which is reflected in the response to the explosive compound of interest. A chloride ion source (methylene chloride permeation tube) was added to the system to stabilize the reactant ions. Typically, chloride reactant ion chemistry is used to increase sensitivity to specific explosive species, such as RDX. In this application, sensitivity of the IMS is not an issue and the chloride was used mainly to stabilize the reactant ion peak behavior and to hopefully suppress the effect of acetone on explosive response over varying injection volume sizes. The temporary solution to the problem has been to use a consistent injection volume, in our case, $2 \mu \mathrm{L}$. Some IMS systems rely upon pre-evaporation of the solvent before sample introduction into the IMS. Direct solution injection was used in this case to increase throughput, reduce cross contamination potential, and simplify the methodology. Good calibration curves were developed using the constant volume injection method for 3 to 5 different standards (Figure 1). It became apparent during the investigation that high levels of non-target explosives present in the same samples can detrimentally effect the response to a target explosive. This interference effect can be attributed to ion chemistry complications, such as competition for charge among analytes.

To simplify the problem, we picked a set of eight samples (in duplicate) containing a wide range of TNT and generally free of potentially interfering other explosives (Location 1). Samples were then diluted as needed for analysis. After an initial run, the samples were all rerun using improved procedures and dilution estimates from the first run. The time consuming portion of the analysis procedure was making the sample dilutions which ranged from 1111:1 to 500,000:1. IMS analysis was quick step with the 15 point calibration curve and triplicate injections of all 16 samples being completed in 1.5 hours.

The results comparing IMS with Method 8330 data are shown in Figures 3 and 4, on linear and log scales respectively. For comparison, EnSys colorimetric screening data as run by Jenkins et al. (1996) are also compared to Method 8330 data (Figures 5 and 6). The ideal the regression line relating 
screening and 8330 data should have an intercept to 0 and a slope of 1 . For the IMS data, the slope is 0.99 and the intercept is -351 . For EnSys, the slope was 0.78 with an intercept of 833 . While the IMS model is closer to the ideal, the spread of data is greater then with EnSys. The correlation coefficient for IMS-was 0.84 and for EnSys was 0.96.

\section{CONCLUSIONS}

These results demonstrate that IMS can be used to rapidly quantify TNT in soil samples free of other explosives and presumably could be effectively used to look at many other individual compounds just as effectively. IMS can also be used to easily detect the presence of a variety of explosives (excluding HMX) but we have yet to demonstrate the capability of simultaneously quantifying multiple explosives. This should be possible with improved software, installation of a short chromatographic column or a programmed injection/desorption front end to separate compounds. If this problem can be overcome, IMS should develop as a very rapid site characterization tool for quantifying multiple individual compounds at very low per sample cost. The main limitation of the technique would be instrument cost and operator training. We intend to continue laboratory development and evaluation of the technique and if these results are positive, conduct field evaluations as well.

\section{ACKNOWLEDGMENT}

The INEL authors thank the U.S. Army Environmental Center and Dr. Thomas Jenkins at CRREL for providing the samples and the EPA Method 8330 analytical results used in this study. This work performed under the auspices of the U.S. Department of Energy, Office Contract No. DE-AC0794ID13223.

\section{REFERENCES}

Avolio, J., R. DeBono, and P. Radwanski., "Ion Mobility Spectrometry (IMS) Field Screening Methods and Analysis of Explosives in Contaminated Soils", in Field Screening Methods for Hazardous Wastes and Toxic Chemicals, VIP-47; Air \& Waste Management Association: Pittsburgh, PA, 2:1037, 1988. only.

Jenkins, T.F., C.L. Grant, G.S. Brar, et. al., Assessment of Sampling Error Associated with Collection and Analysis of Soil Samples at Explosive Contaminated Sites (Phase 1): Status Report, Special Report 96-15, U.S. Army Corps of Engineers, Cold Regions Research and Engineering Laboratory, Hanover, NH, 1996.

Rodacy, P. and P. Leslie, Ion Mobility Spectroscopy as a Means of Detecting Explosives in Soil Samples, Sand-92-1522C, Sandia National Laboratories, Albuquerque, NM, 1992.

\section{DISCLAIMER}

This report was prepared as an account of work sponsored by an agency of the United States Government. Neither the United States Government nor any agency thereof, nor any of their employees, makes any warranty, express or implied, or assumes any legal liability or responsibility for the accuracy, completeness, or usefulness of any information, apparatus, product, or process disclosed, or represents that its use would not infringe privately owned rights. Reference herein to any specific commercial product, process, or service by trade name, trademark, manufacturer, or otherwise does not necessarily constitute or imply its endorsement, recommendation, or favoring by the United States Government or any agency thereof. The views and opinions of authors expressed herein do not necessarily state or reflect those of the United States Government or any agency thereof. 


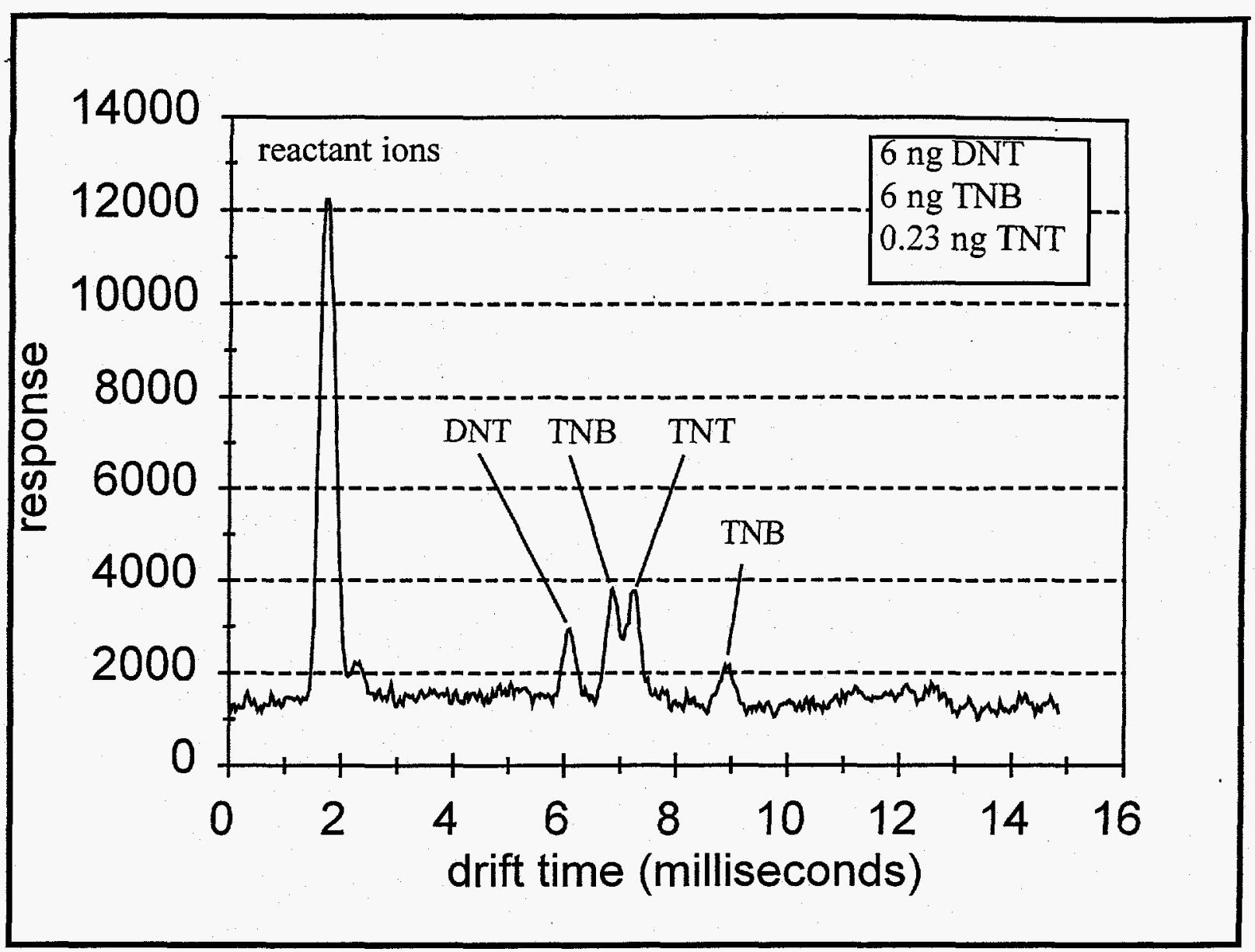

Figure 1. Ion mobility spectrum of explosive mixture in acetone. 


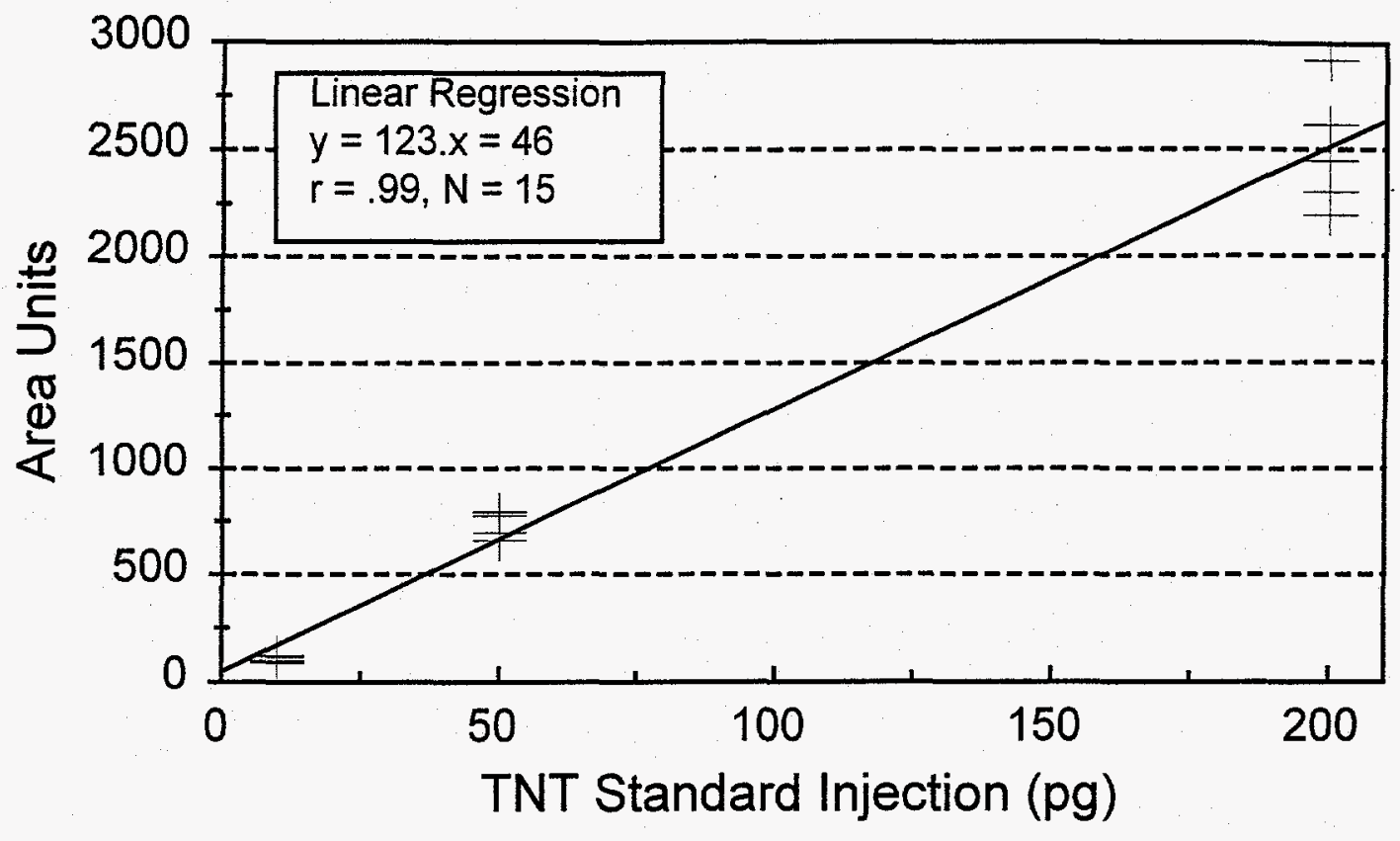

Figure 2. IMS calibration curve - TNT 


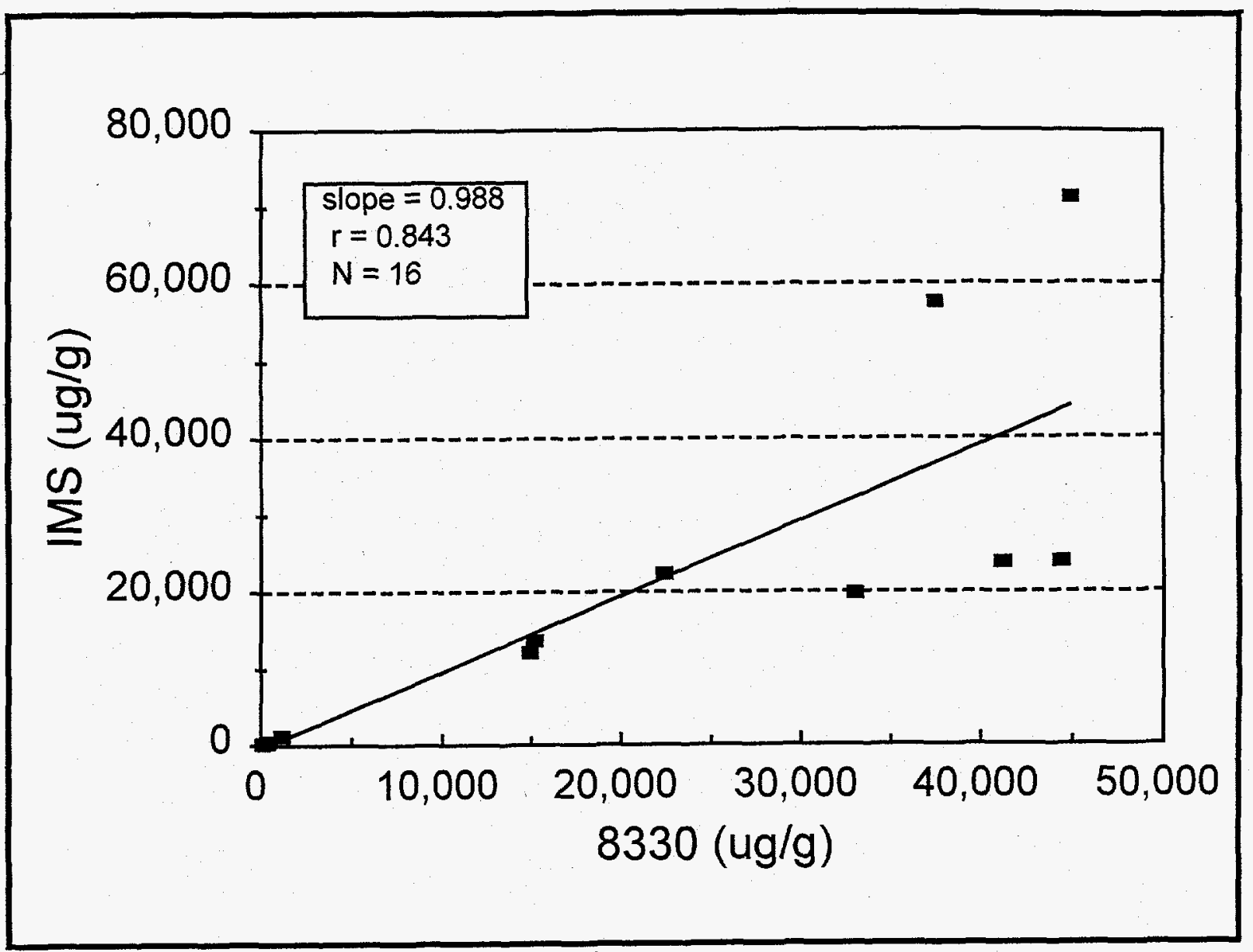

Figure 3. IMS/EPA 8330 comparison - site L1, TNT 


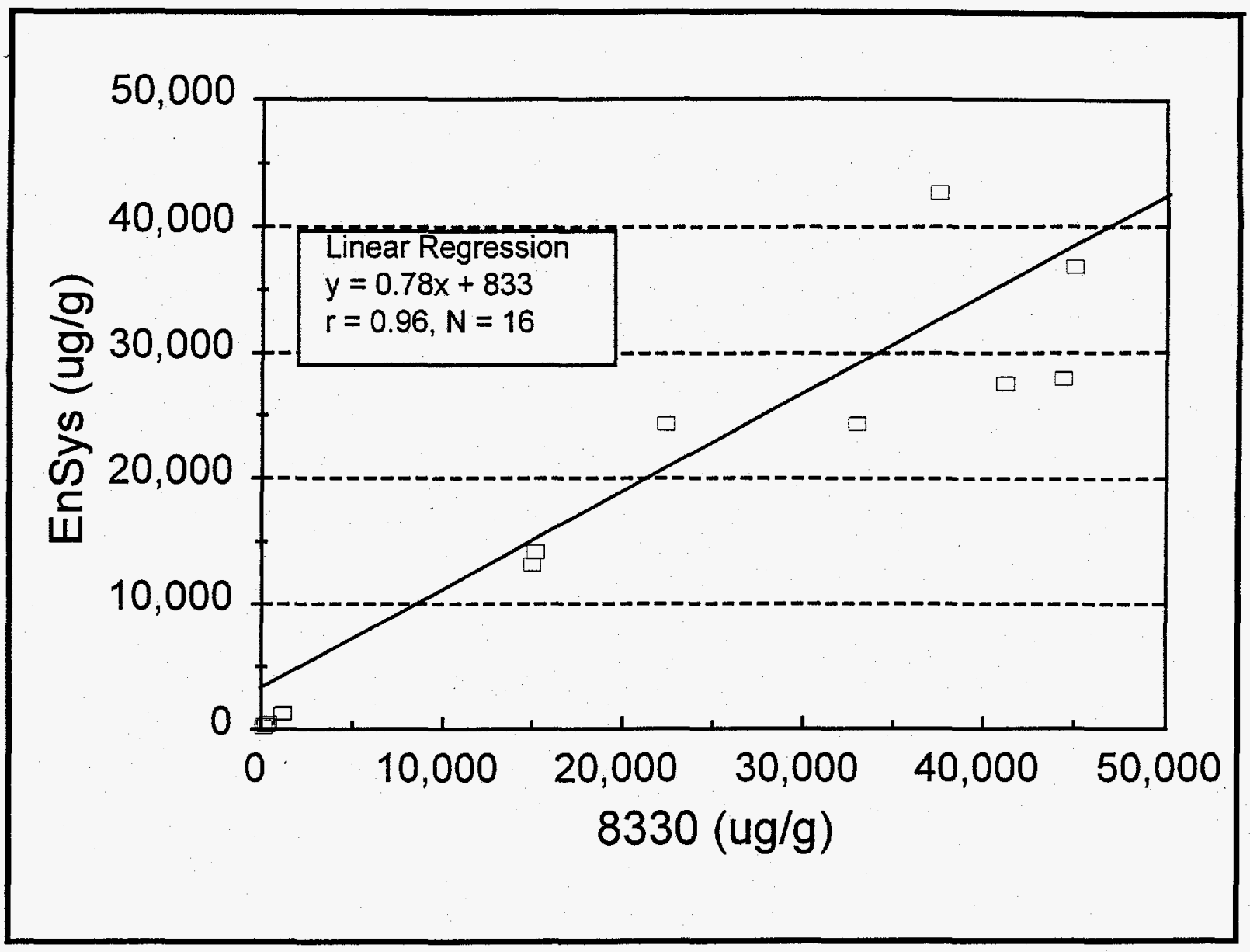

Figure 4. EnSys/EPA 8330 comparison - site L1, TNT 


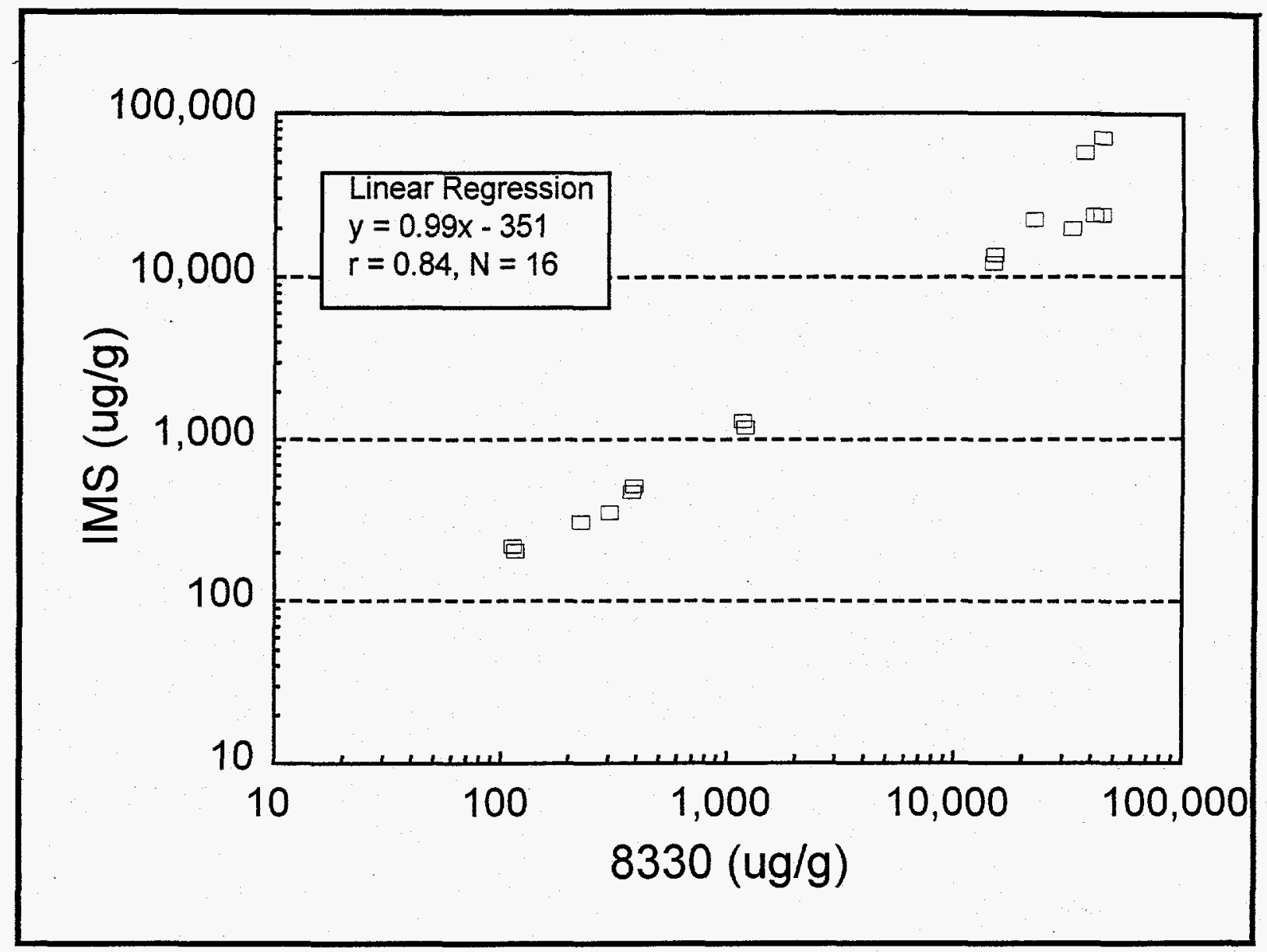

Figure 5. IMS/EPA 8330 logarithmic comparison - site L1, TNT 


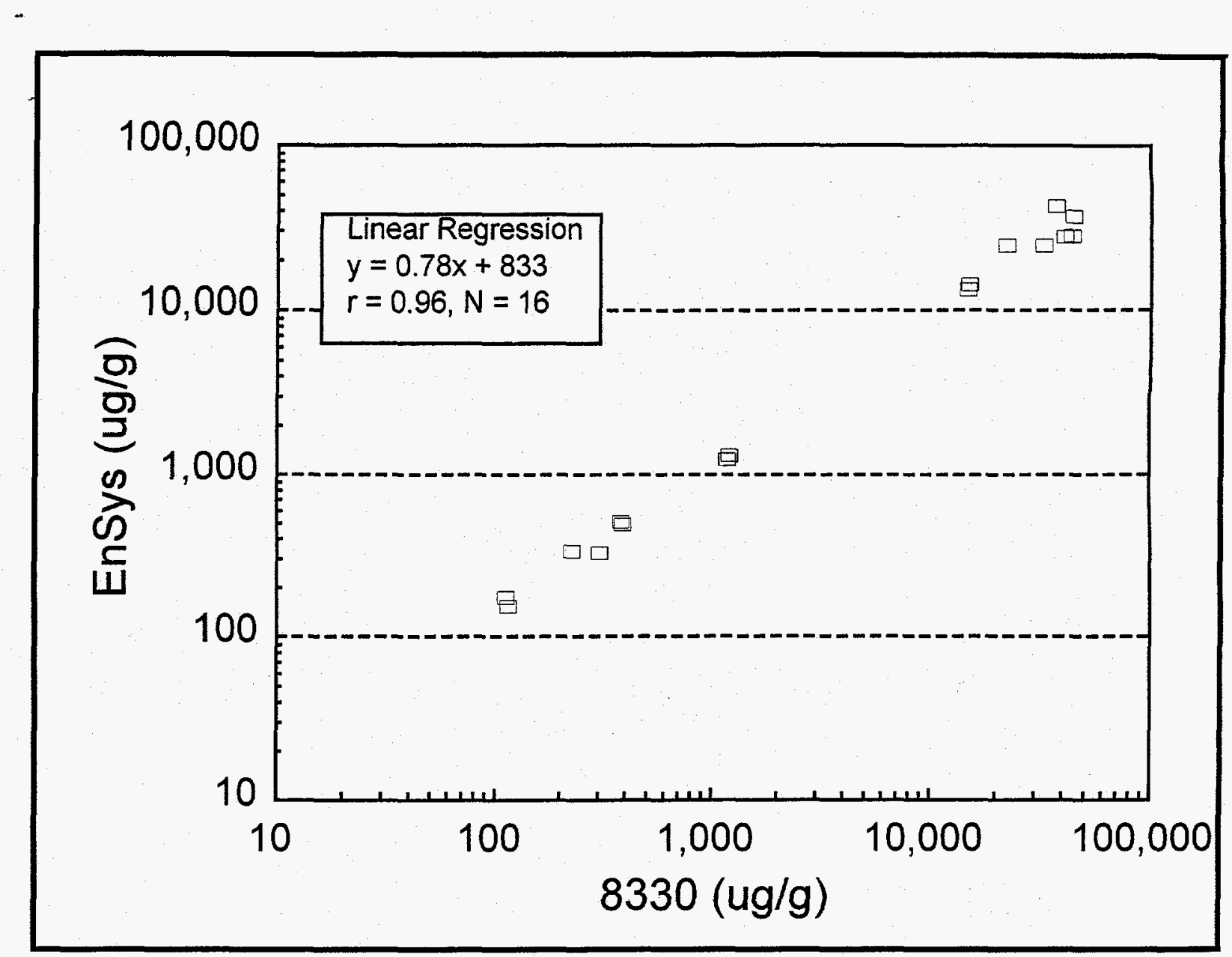

Figure 6. EnSys/EPA 8330 logarithmic comparison - site L1, TNT 